\title{
Summary of PHENIX Transverse Spin Physics Results
}

\author{
K.Oleg Eyser on behalf of the PHENIX collaboration \\ University of California, \\ Riverside, CA 92521, USA
}

\begin{abstract}
The PHENIX experiment at the Relativistic Heavy Ion Collider (RHIC) has accumulated a substantial data set of transversely polarized protons over the last few years. Measurements include inclusive single spin asymmetries at mid-rapidities at $\sqrt{s}=200$ GeV. In 2006, new data sets of $2.7 \mathrm{pb}^{-1}$ at $\sqrt{s}=200 \mathrm{GeV}$ and $20 \mathrm{nb}^{-1}$ at $\sqrt{s}=$ $62.4 \mathrm{GeV}$ have been taken. Along with a new calorimeter at forward rapidities, a variety of asymmetries and probes has been proposed, which may help to disentangle the contributions from the theoreatical model calculations. We present the status of the PHENIX measurements, and how they can help lead to a deeper understanding of the transverse proton spin structure.
\end{abstract}

\section{Introduction}

Large single-spin asymmetries have been observed in different high energy $p+p$ collisions in the past [2], although they were expected to vanish in perturbative QCD calculations at large transverse momenta $p_{T}$. The spin structure of the nucleon includes a term called transversity distribution $\delta q$, which is different from the longitudinal quark distribution $\Delta q$ because relativistic boosts and rotations do not commute. Transversity therefore probes the relativistic nature of the nucleon and can be determined from transversely polarized scattering processes

$$
A_{T T} \propto(\delta q)^{2}, \quad A_{N} \propto \delta q .
$$

The analyzing power $A_{N}$ poses a direct measure of transversity in combination with another chiral odd function. Proposed models include spin dependent fragmentation functions (Collins effect, [3]), asymmetries in the intrinsic transverse momentum distributions of partons (Sivers effect, [4]), and quark gluon field interference in higher twist calculations [5]. The latter might have a major impact in the investigated energy regime, especially at lower $p_{T}$.

\section{The PHENIX Experiment}

The PHENIX experiment uses a multi-purpose detector with a high bandwidth data acquisition system and multiple triggers. The central arms cover -0.3 to 0.3 in pseudo-rapidity $\eta$ and two times $\pi / 2$ in azimuthal angle. Charged particles are tracked in a drift chamber (DC) outside of a central axial magnetic field and followed by several layers of pad chambers (PC1 through PC3). A ring imaging Cerenkov detector (RICH) allows particle separation of pions and electrons below the threshold momentum of $4.9 \mathrm{GeV} / \mathrm{c}$. Additionally, the particle energies are measured in $\mathrm{PbSc}$ and $\mathrm{PbGl}$ electro-magnetic calorimeters (EMCAL).

The two muon arms cover the full azimuth at $1.2<|\eta|<2.4$. Each arm consists of three tracker stations (MuTr) followed by muon identification detectors (MuID) sandwiched between steel absorbers.

Also, there are two sets of global detectors, the beam-beam-counters (BBC) and the zero degree calorimeter (ZDC), which mainly serve trigger and monitoring purposes. In 2006, a 
new $\mathrm{PbWO}_{4}$ calorimeter was commissioned in the south hemisphere of the experiment in forward direction $(3.2<\eta<3.8)$ with full azimuthal coverage.

\section{$3 \quad$ Asymmetries}

Transverse single spin asymmetries are described by:

$$
\epsilon=A_{N} \cdot P \cdot\left\langle\cos \varphi>=\frac{N^{+}-N^{-}}{N^{+}+N^{-}}\right.
$$

where the combination of the polarization $P$ and the analyzing power $A_{N}$ can lead to an azimuthal modulation of the differential cross section. Since this asymmetry is maximum perpendicular to the polarization vector and vanishes in its direction, for extended detectors, the cosine-modulation has to be taken into account. The yields $N^{+}$and $N^{-}$refer to two different polarization states, which in this case point in the direction of $\varphi=0.5 \cdot \pi$ and $\varphi=1.5 \cdot \pi$.

Asymmetries between the left and right side hemispheres of the detectors are considered to be simple asymmetries, as are pure polarization asymmetries in one detector, i.e. asymmetries between two polarization states $P^{\uparrow}=+P$ and $P^{\downarrow}=-P$. These asymmetries are susceptible to acceptance, efficiency, and luminosity differences of the respective yields. Therefore, it is usually better to use geometric means of the yields and thereby removing those effects in first order:

$$
A_{N}=\frac{1}{P} \cdot \frac{\sqrt{N_{\text {left }}^{\uparrow} \cdot N_{\text {right }}^{\downarrow}}-\sqrt{N_{\text {left }}^{\downarrow} \cdot N_{\text {right }}^{\uparrow}}}{\sqrt{N_{\text {left }}^{\uparrow} \cdot N_{\text {right }}^{\downarrow}}+\sqrt{N_{\text {left }}^{\downarrow} \cdot N_{\text {right }}^{\uparrow}}} .
$$

\section{Results}

PHENIX has measured inclusive neutral pion and charged hadron cross sections and single spin asymmetries in the central region previously [6]. Cross sections have been shown to be in good agreement with perturbative QCD calculations. Asymmetries are consistent with zero and have been utilized to constrain the gluon Sivers function [7].

Forward neutron asymmetries have been measured in the ZDC in forward and backward hemispheres. These asymmetries vanish at negative $x_{F}$ and rise to about $8 \%$ at positive $x_{F}$ as expected from RHIC-IP12 [8]. From the azimuthal distributions, one can determine the alignment of the polarization vector. Forward neutron asymmetries are, therefore, utilized as a monitoring tool for the spin rotator commissioning.

Recent measurements from 2006 include a first glimpse into heavy flavour production. Since heavy quarks are expected to be predominantly produced by gluon fusion and gluons do not exhibit transversity, the Collins mechanism should be minimized. Measured single spin asymmetries could then be more easily connected to gluon (or less prominently quark) Sivers function. The $J / \Psi$ di-muon decay is detected in the muon arms, see figure 1 . Several checks of background asymmetries have been carried out, including same-sign di-muon spectra and sidebands around the $J / \Psi$-peak. So far, the asymmetries are consistent with zero. 

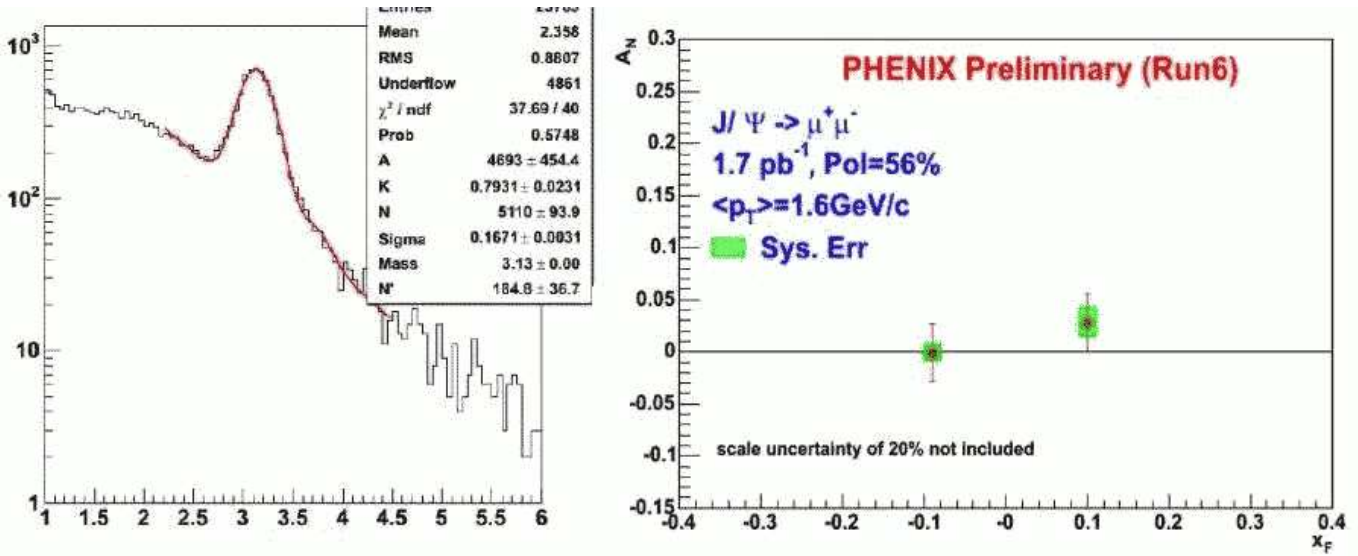

Figure 1: $J / \Psi \rightarrow \mu \mu$ : mass spectrum with di-muon peak at $3 \mathrm{GeV}$. Single spin asymmetry from forward and backward directions (positive and negative $x_{F}$ ).

\section{Outlook}

A new electro-magnetic calorimeter has been installed in the south detector arm prior to the 2006 RHIC run. After commissioning, a data set of $20 \mathrm{nb}^{-1}$ could be recorded at $\sqrt{s}=62.4 \mathrm{GeV}$. While the analysis is still on-going, the detector calibration was finished in early 2007 and first pion asymmetries of a few percent have been seen and seem to follow the general behaviour of those seen by BRAHMS and STAR. These asymmetries can additionally be used for the monitoring of the polarization vector, see figure 2 . The figure is exemplary and contains only part of the data set. The beam polarization is not yet considered in the asymmetries.

Another proposed way to directly access the Sivers function is to look for asymmetries in two-jet back-to-back correlations [9]. In this correlation, the Collins effect should average out and not contribute to any asymmetry. For PHENIX, the jet prediction has been smeared out in order to transfer it to two-hadron correlations. A first proof-of-principle analysis has been done with 2005 data with only a residual transverse component in the longitudinal polarization.

Transversity on the other hand might be directly probed via interference fragmentation functions. A Sivers function would not contribute to a correlation asymmetry between two hadrons, this time coming from the same fragmentation process. Such an analysis depends on the knowledge of the interference fragmentation functions, which have to be extracted from other experiments, e.g. Belle.

\section{Summary}

PHENIX has measured transverse single spin asymmetries of inclusive neutral pions and charged hadrons at mid-rapidities in the past. Forward neutron asymmetries are seen and utilized for monitoring of the polarization vector in the interaction region. New analyses include heavy flavours via $J / \Psi$ production with its di-muon decay. First neutral pion asymmetries have been seen at large rapidities, which generally show the same behaviour of the 

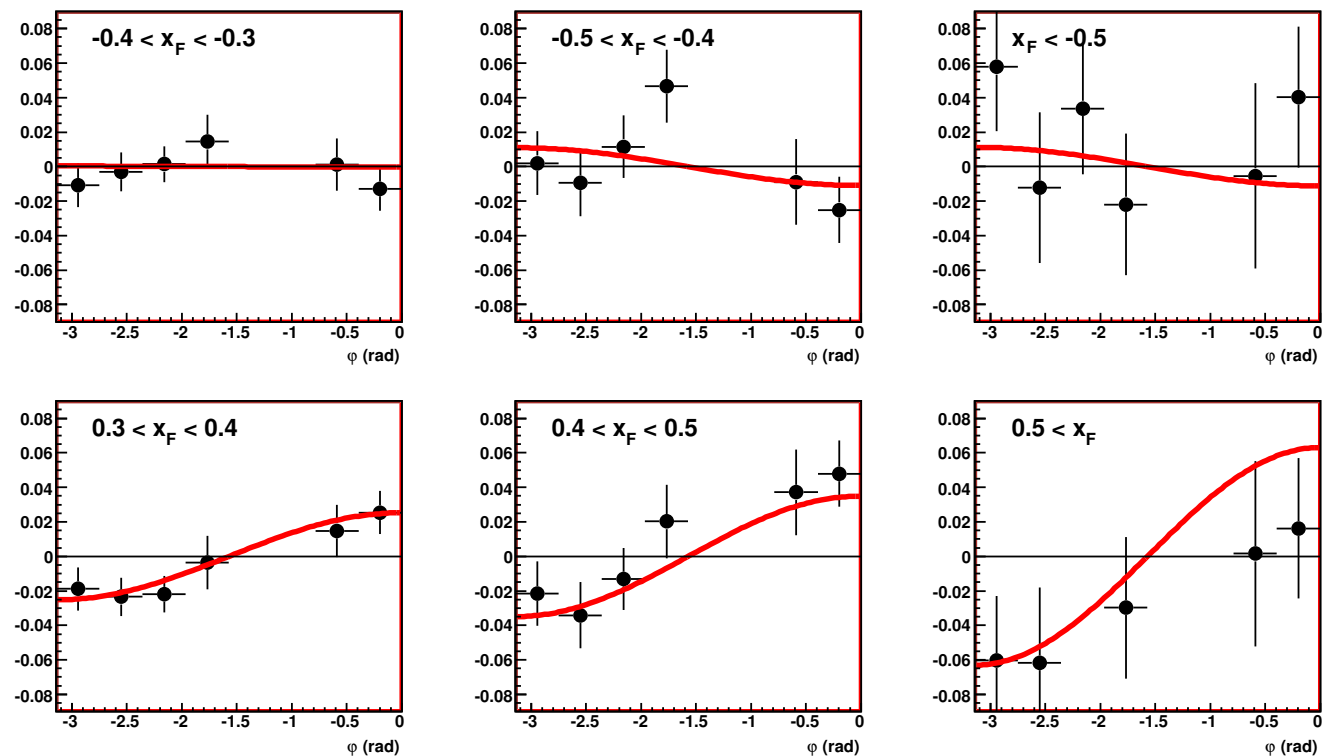

Figure 2: Raw neutral pion single spin asymmetries at large rapidities $(3.2<\eta<3.8)$. The asymmetries are calculated with geometric means of yields, see above, and fitted with a cosine function. The polarization has not yet been included.

pion asymmetries seen in BRAHMS and STAR. Future analyses will include two-hadron correlations to probe the Sivers function and directly access transversity.

\section{References}

[1] Slides: http: //indico. cern. ch/contributionDisplay . py? contribId=156\&sessionId=4\&conf Id=9499

[2] D.L. Adams et al., Phys. Lett. B264 462 (1991);

J. Adams et al., Phys. Rev. Lett. 92171801 (2004)

[3] J. Collins, Nucl. Phys. B396 161 (1993).

[4] D. Sivers, Phys. Rev. D 43261 (1991).

[5] Y. Koike, "Single transverse-spin asymmetry in $\mathrm{p} \mathrm{p}($ pol. $)$ - $i$ pi X and e p(pol.) - $i$ pi [arXiv:hep$\mathrm{ph} / 0210396]$

[6] S. Adler et al., Phys. Rev. Lett. 95202001 (2005).

[7] M. Anselmino et al., Phys. Rev. D 74094011 (2006).

[8] A. Bazilevsky et al., Phys. Lett. B 650 (2007) 325 [arXiv:hep-ex/0610030].

[9] C.J. Bomhof et al., Phys. Rev. D 75 (2007) 074019 [arXiv:hep-ph/0701277]; D. Boer and W. Vogelsang, Phys. Rev. D 69, 094025 (2004) [arXiv:hep-ph/0312320]. 